\section{BMJ Global Health}

\title{
Not just a number: examining coverage and content of antenatal care in low- income and middle-income countries
}

\author{
Lenka Benova, ${ }^{1}$ Özge Tunçalp, ${ }^{2}$ Allisyn C Moran, ${ }^{3}$ Oona Maeve Renee Campbell ${ }^{1}$
}

To cite: Benova L, Tunçalp 0̈, Moran AC, et al. Not just a number: examining coverage and content of antenatal care in low-income and middle-income countries. BMJ Glob Health 2018;3:e000779. doi:10.1136/ bmjgh-2018-000779

Handling editor Sanni Yaya

Received 14 February 2018 Revised 19 March 2018 Accepted 20 March 2018
Check for updates

${ }^{1}$ Faculty of Epidemiology and Population Health, London School of Hygiene \& Tropical Medicine, London, UK ${ }^{2}$ Department of Reproductive Health and Research, WHO, Geneva, Switzerland ${ }^{3}$ Department of Maternal, Newborn, Child and Adolescent Health, WHO, Geneva, Switzerland

Correspondence to Dr Lenka Benova; lenka.benova@Ishtm.ac.uk

\section{ABSTRACT}

Introduction Antenatal care (ANC) provides a critical opportunity for women and babies to benefit from goodquality maternal care. Using 10 countries as an illustrative analysis, we described ANC coverage (number of visits and timing of first visit) and operationalised indicators for content of care as available in population surveys, and examined how these two approaches are related. Methods We used the most recent Demographic and Health Survey to analyse ANC related to women's most recent live birth up to 3 years preceding the survey. Content of care was assessed using six components routinely measured across all countries, and a further one to eight additional country-specific components. We estimated the percentage of women in need of ANC, and using ANC, who received each component, the six routine components and all components.

Results In all 10 countries, the majority of women in need of ANC reported 1+ ANC visits and over two-fifths reported 4+ visits. Receipt of the six routine components varied widely; blood pressure measurement was the most commonly reported component, and urine test and information on complications the least. Among the subset of women starting ANC in the first trimester and receiving $4+$ visits, the percentage receiving all six routinely measured ANC components was low, ranging from $10 \%$ (Jordan) to around 50\% in Nigeria, Nepal, Colombia and Haiti.

Conclusion Our findings suggest that even among women with patterns of care that complied with global recommendations, the content of care was poor. Efficient and effective action to improve care quality relies on development of suitable content of care indicators.

\section{INTRODUCTION}

Maternal and perinatal morbidity and mortality remain high, especially in low-income, middle-income countries (LMICs). In 2015, an estimated 303000 women died from pregnancy-related causes, 2.6 million babies were stillborn (half in the third trimester) and 2.7 million newborns died in the first month of life. ${ }^{12}$ Reducing this burden of ill health has been prioritised in the United Nations' Global Strategy for Women's, Children's, and Adolescent's Health (2016-2030), with ambitious mortality reduction targets

\section{Key questions}

\section{What is already known?}

- Antenatal care (ANC) is of crucial importance to the health of pregnant women and their babies, including through linkage to childbirth and postnatal care.

- Currently, ANC coverage among pregnant women in low-income, middle-income countries (LMICs) is captured through indicators of number of visits $(1+$ and $4+)$ and timing of the first visit.

- Several recent publications have highlighted issues with content and quality of ANC in these settings.

\section{What are the new findings?}

- Our results show that most women in the selected 10 countries received some ANC, although patterns of coverage, particularly the mean number of visits, varied greatly by country.

- However, the self-reported content of care was suboptimal even among women meeting recommendations on number of ANC visits and timing of first visit at the time of survey.

\section{What do the new findings imply?}

- This study adds to a body of evidence highlighting the importance of ensuring content and quality of care among the rising proportion of women in LMICs making contact with ANC providers, which is crucial for reducing the mortality and morbidity associated with pregnancy and childbirth.

- We also call on improving measurement of quality of ANC that can guide further improvements in provision.

included in the Sustainable Development Goals (SDGs). ${ }^{34}$ There are 210 million pregnancies occurring each year, and with goodquality care, including during antenatal care (ANC), the majority of adverse maternal and perinatal outcomes can be prevented. ${ }^{56}$

In November 2016, WHO released new comprehensive recommendations on routine ANC for pregnant women, including adolescent girls. ${ }^{7}$ This guideline adopted a human rights based approach to respond to the complex nature of $\mathrm{ANC}$ practice, 


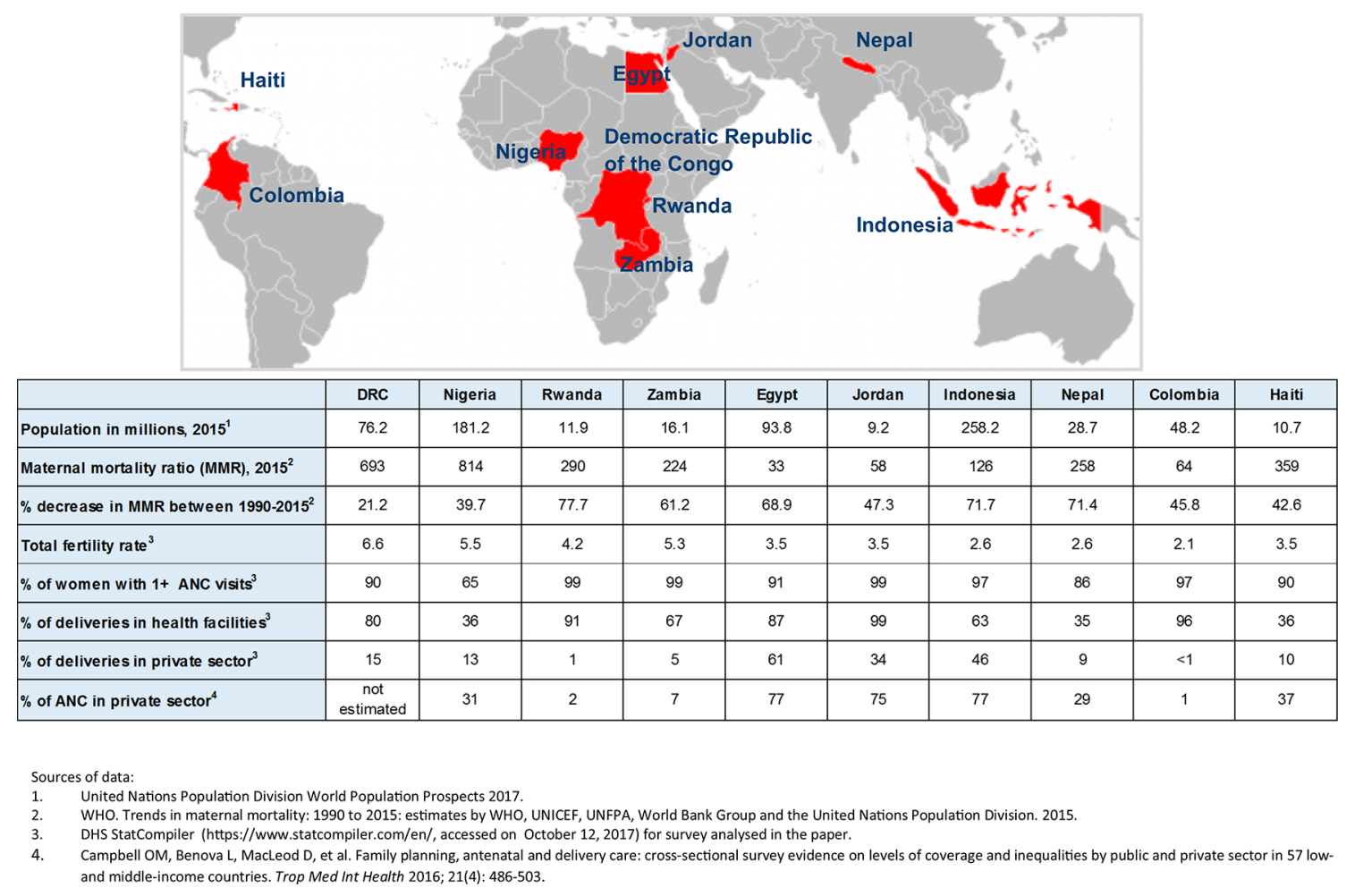

Figure 1 Included countries and selected population indicators. ANC, antenatal care; DRC, Democratic Republic of Congo.

organisation and delivery within diverse health systems. It focused on person-centred care and well-being, and aimed to prevent death and ill-health. This ANC guideline includes 49 recommendations: 14 nutritional interventions, 8 maternal and fetal assessments, 5 preventive measures, 6 interventions for common physiological symptoms, 6 health systems interventions and 10 routine recommendations from other WHO guidelines. ${ }^{7}$ Further, the new guideline recommends eight ANC contacts, with the first contact in the first trimester (up to 12 weeks of gestation) and increased contacts (five) during the third trimester, the time of highest risk for major maternal complications such as pre-eclampsia/eclampsia. Routine ANC has universal components applicable to every woman, but overall the recommendations are designed to be adaptable so that countries with different burdens of disease, social and economic situations, and health system structures can implement the recommendations based on their context and their populations' needs. Five of the 49 recommendations only apply in a research context.

ANC coverage can be estimated via routine health information systems, but in LMICs, it is predominately captured via population-based surveys such as the Demographic and Health Surveys (DHS) and Multiple Indicator Cluster Survey. Global monitoring of the Millennium Development Goals (MDGs) included indicators on the number of ANC visits $(1+$ and $4+) .{ }^{8}$ Coverage of $4+$ ANC visits is also 1 of 12 core maternal health indicators for global monitoring and reporting for the SDGs. ${ }^{9}$ However, the number of visits does not provide information on the content of care received in these visits, and was identified as a priority area for indicator development. ${ }^{9}$ Hodgins and D'Agostino (2014) pointed out that these coverage indicators capture contacts with care providers; instead they called for indicators of effective ANC coverage, by focusing on the content of care received, in order to begin to capture quality of care, an approach conceptually endorsed by $\mathrm{Ng}$ and colleagues. ${ }^{1011}$ The 2016 WHO guideline also emphasises the importance of quality of care during each contact, highlighting components of essential content of high-quality care. There is a need to develop monitoring indicators which focus on the content of ANC as part of quality of care.

The objective of our study is to: (1) Describe ANC coverage using the MDG-era indicators of number of visits $(1+$ and $4+)$ in selected countries. (2) Operationalise and examine and timing of the first visit and indicators for content of care based on WHO ANC guidelines, as available in multicountry DHS. (3) Examine the extent to which these measures relate to the MDG-era ANC indicators.

\section{METHODS \\ Data}

DHS are cross-sectional nationally representative household surveys, usually covering 5000 to 30000 households. Standard model questionnaires are used but can be adapted by each country; optional modules can also be added. The sampling design is a multilevel cluster survey, which often oversamples certain areas; individual women's survey weights are needed in analysis to adjust for this and for non-response. Respondents are either 


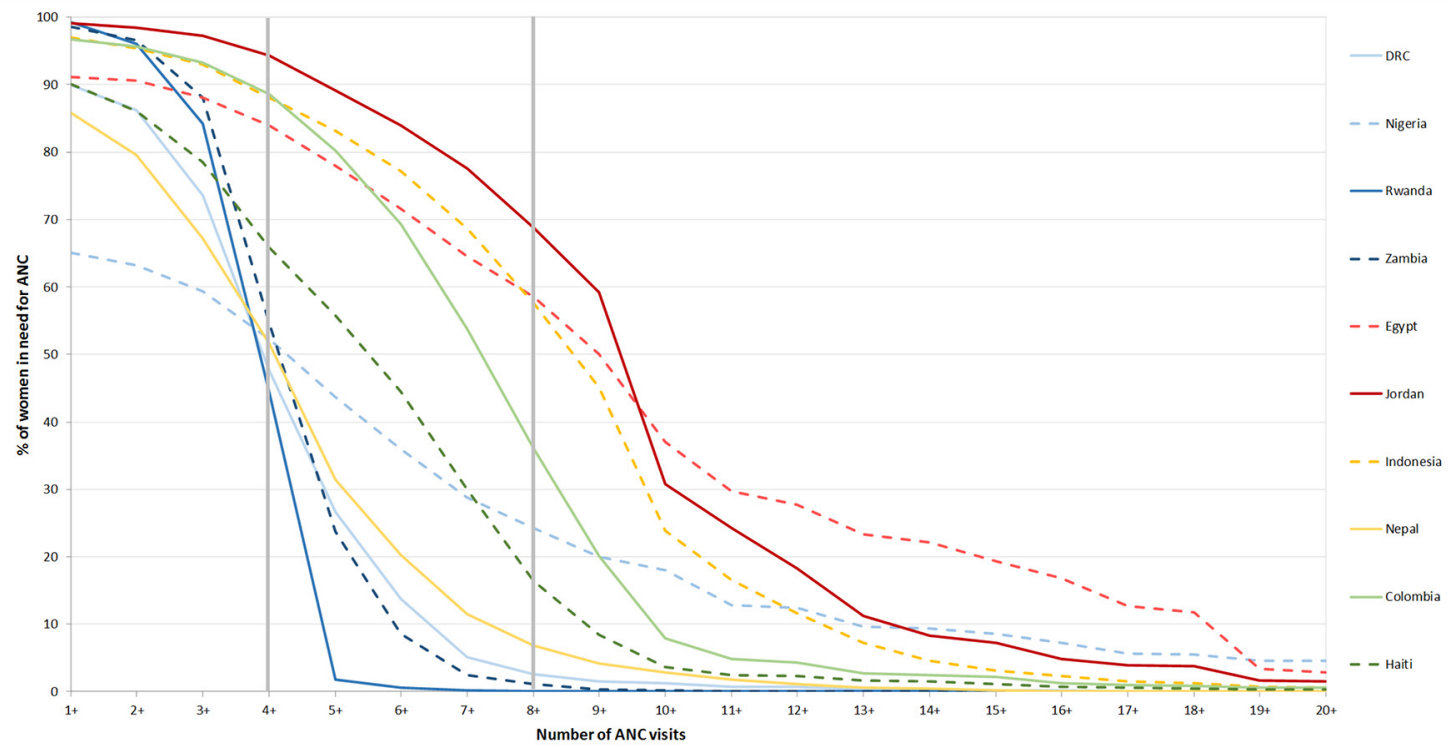

Figure 2 Distribution of cumulative number of antenatal care (ANC) visits among women with a live birth, by country. DRC, Democratic Republic of Congo.

ever-married or all women of reproductive age (15-49 years). Data are generally based on women's self-reports, and questions on ANC are asked for the most recent pregnancy, ending in live birth, in a specified recall period.

We used the most recently available survey data (as of November 2016) for 10 LMICs with varying disease burden patters and health systems from four world regions: sub-Saharan Africa (Democratic Republic of Congo (DRC), Nigeria, Rwanda, Zambia), the Middle East (Jordan, Egypt), South/South-East Asia (Nepal, Indonesia), and Latin America and the Caribbean (Haiti, Colombia). We selected countries purposively to cover four geographical regions and a range of achievements in meeting the MDGs for maternal mortality reduction (figure 1). ${ }^{1}$ Previous research has shown that such a selective case study approach can be effective in highlighting a range of situations while allowing for consideration of context-specific issues in analysis and interpretation. ${ }^{12}$

\section{Population}

We analysed ANC use and content of care among women who needed ANC, which are those who had a live birth in the 3 years (0-36 months) preceding the survey (except in Colombia where survey recall period was 1 year). ANC for the most recent live birth was analysed.

\section{Definitions}

Women were asked about whether they saw anyone for ANC during the pregnancy preceding their most recent live birth, and if they did, who they saw for this care (type of person/health professional cadre), where they received the care (with multiple responses to providers/ locations allowed), how many months pregnant women were when they first received ANC, and how many times they received ANC during the pregnancy. Next, they were asked about whether they received specific ANC components (yes/no) during any ANC over the course of their entire pregnancy,

We defined use of ANC as reporting a non-zero number of ANC visits or a non-missing location of ANC provision or a valid type of professional who provided ANC. Since both the timing and number of visits were indicators on which we disaggregated care components, we excluded from analysis ANC users who had a missing number of visits or missing timing of first visit. A small percentage of women in the sample $(1.25 \%)$ reported $>30$ ANC visits during pregnancy; many of these values are likely to be data recording or data entry errors; these values were recoded as missing and therefore excluded from analysis. The years of survey, extent of missingness and final analysis sample sizes are provided in online supplementary material 1.

We used the number of visits as a continuous variable and created categories based on the previous four-visit ANC model ${ }^{13}$ and 2016 WHO guideline recommending eight contacts: one to three, four to seven, and eight or more. We also reported on all ANC users (women with $1+$ visit) and women with $4+$ visits (this included women with $8+$ visits). The timing of the women's first ANC visit was measured in the DHS in months, and categorised, based on WHO recommendations, as occurring in the first trimester of pregnancy (months 1-3), or in the second or third trimester (month 4 of pregnancy or later). We also report on categories according to combinations of number of visits and timing of the first visit.

Women who reported receiving ANC were asked whether they received specific care components during their pregnancy (eg, 'During any of your antenatal care visits, were you told about things to look out for that might suggest problems with the pregnancy?'). Women were not asked how many times they received each care 


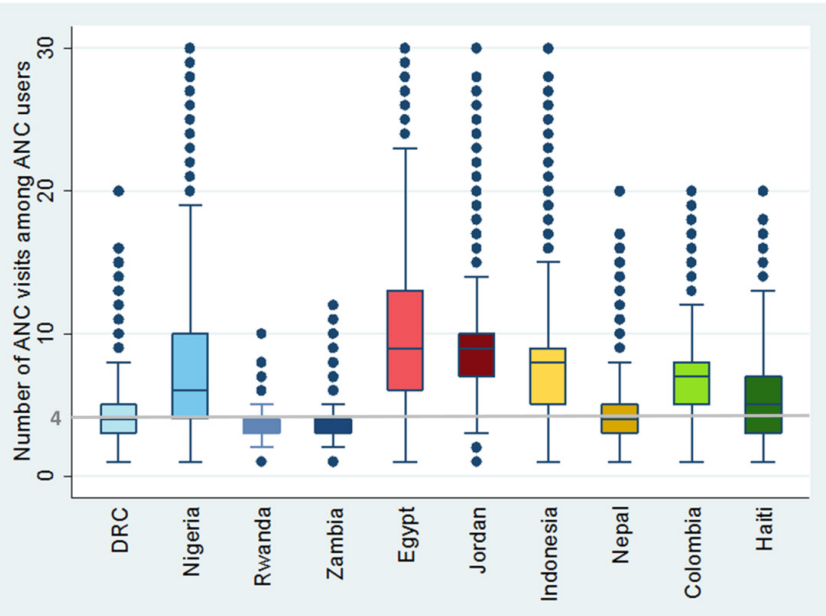

Figure 3 Box plot of number of antenatal care (ANC) visits among ANC users, by country. DRC, Democratic Republic of Congo.

component, during which visits, with which provider or location, or at what gestation during their pregnancy, with the exception of tetanus injections (asked number and timing of injections across pregnancies) and iron/ folate supplementation (asked number of pills). Each country collected information on a slightly different set of ANC care components, and some were asked in slightly different ways. We assessed content of care for three sets of care components: (1) All six components routinely measured across all included countries on the DHS, including blood pressure measured, urine sample taken, blood sample taken, tetanus protection, iron supplementation and receipt of information on potential complications (from now on referred to as 'routine' ANC components). (2) All further components measured that were specific to each country, such as having been weighed and receiving intermittent preventive treatment for malaria ('country-specific' components). (3) All components measured (combination of first two, 'all components' for short). Across the countries, between 1 and 8 country-specific ANC components were captured in addition to the routine, a total of 25 unique components. We excluded receipt of intermittent preventive treatment for malaria in Colombia and intestinal parasite prophylaxis in Egypt as these were not part of their national ANC guidelines, and their coverage was extremely low. The wording of the questions on components of ANC on the DHS questionnaire for each country is shown in online supplementary material 2, together with a mapping of the three sets of components (routine, country-specific and all) onto existing WHO guidelines on ANC care content.

We estimated the percentage of ANC users who reported receiving each care component and the percentage of users who reported receiving all six routine, all country-specific and all measured components. For all routine and all measured components, we also calculated the mean number of components received. Each component carried equal weight (simple average). Missing values in receipt of care components were recoded to 'no' (did not receive care component); this also applied to missingness due to skip patterns (ie, if a question about a care component was not asked because a preceding prerequisite care component was not received). We estimated the percentage and mean number of components in categories with $\geq 50$ weighted observations. The percentages of ANC users receiving the above care components were estimated for all ANC users and for specific categories of number of ANC visits and timing of first ANC visit.

\section{Analysis}

Data analysis was conducted in Stata SE V.15 (College Station, Texas, USA), using the suyset command to account for survey design (sample weights, clustering and stratification).

\section{Ethical approval}

The DHS receive government permission, use informed consent and assure respondents of confidentiality.

\section{RESULTS}

Surveys included in the analysis were collected between 2010 (Colombia) and 2015 (Rwanda). The sample sizes of women with live births ranged from 2857 (Nepal) to 16721 (Nigeria), with low proportions of data missing on number of ANC visits and timing of the first visit (range $0.2 \%-2.6 \%$ among women with any ANC), as shown in supplementary material 1 .

The distribution of the cumulative number of ANC visits among all women with a live birth in the recall period in the 10 countries (women in need of ANC) shows that except in Nigeria, where only $65.1 \%$ reported any ANC visits, most women $(>85 \%)$ who needed ANC received 1+ ANC visits (figure 2). Across the countries, between $44.5 \%$ (Rwanda) and $94.3 \%$ (Jordan) of women in need of ANC reported receiving the recommended $4+\mathrm{ANC}$ visits.

The median numbers of ANC visits among women who received 1+ ANC visits show that women in five countries (DRC, Rwanda, Zambia, Nepal, and Haiti) appeared to have 4 ANC visits (figure 3). Women in the other five countries (Nigeria, Egypt, Jordan, Indonesia and Colombia) showed higher median numbers of ANC visits but achieved them with varying degrees of consistency. This means that in countries such as Egypt and Nigeria, many ANC users did not receive 4+ ANC visits, while a substantial proportion received far more.

Figure 4 shows the distributions of combination categories of number of ANC visits and timing of the first ANC visit, among ANC users. The two darker green shades represent those complying with $\mathrm{WHO}$ recommendations on number and timing of ANC visits at the time of survey. These ranged from $14.9 \%$ in DRC to $89.1 \%$ in Jordan. The two lighter shades of green represent women with the recommended number of visits, but who initiated ANC after the first trimester. The pale grey represents women 


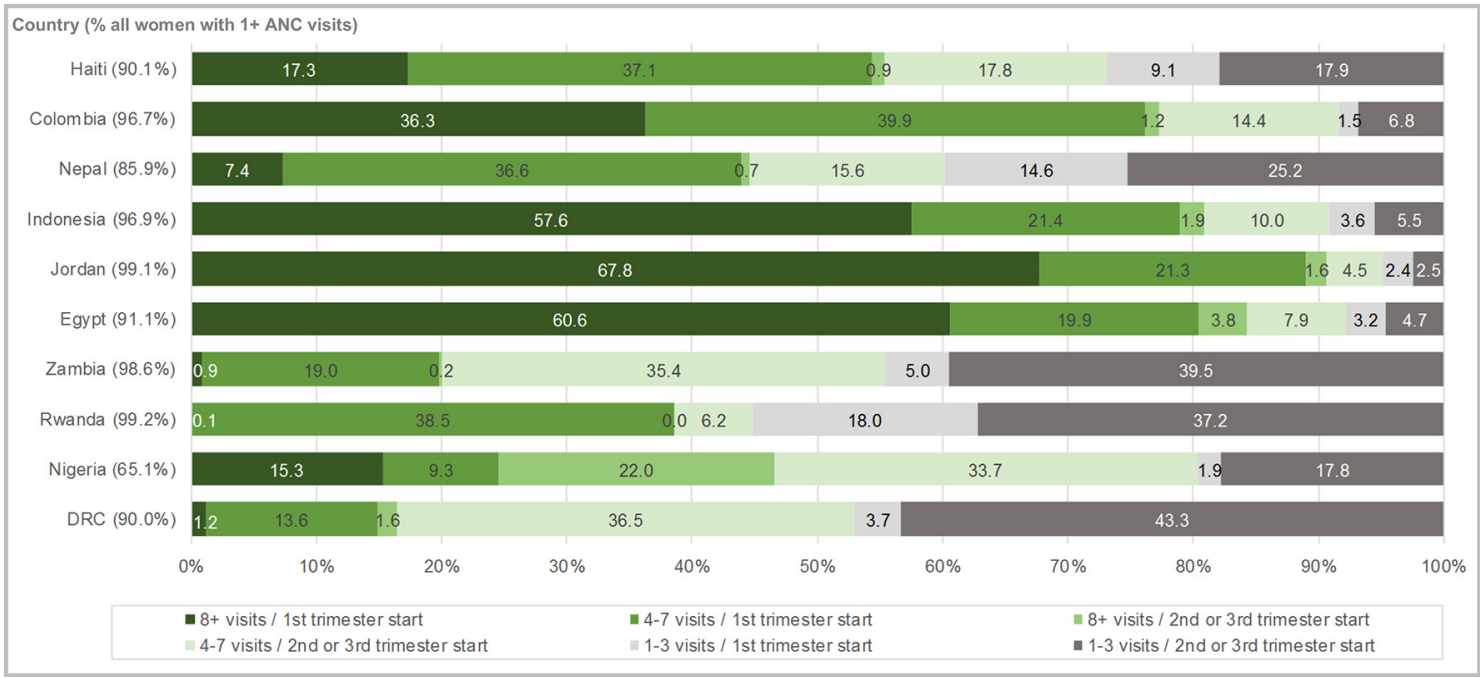

Figure 4 Combination of timing of first visit and number of visits among ANC users, by country. ANC, antenatal care; DRC, Democratic Republic of Congo.

who started ANC in the first trimester, but did not obtain the recommended number of visits. Nepal and Rwanda had the highest percentage in this category $(14.6 \%$ and $18.0 \%$, respectively). However, in both countries, $>45 \%$ of women in this category received exactly three ANC visits and were therefore only one additional visit away from achieving the recommended number of visits and timing of first visit. The dark grey segment represents women who fulfilled neither recommendation, and ranged from $2.5 \%$ in Jordan to $43.3 \%$ in DRC. The measures of ANC content for each country by the indicators of number of timing and visits are shown in supplementary material 3. These tables show the percentage of ANC users who reported receiving each measured ANC care component, the percentage of women who reported receiving all six routine and all measured ANC components, and the mean reported number of the six routine and all available care components per country. Coverage of the individual routine components varied by component and country. For example, among women receiving $4+$ visits, and starting in the first trimester, blood pressure measurement (>80\% in all 10 countries), iron supplementation and blood test tended to be high in most countries ( $>80 \%$ in 8 and 7 of the 10 countries, respectively). Conversely, receipt of information on potential complications among this category of users was $>80 \%$ in only four countries (Rwanda, Zambia, Colombia, Nepal).

The percentages of women who reported receiving all six routine components varied by country and by number of visits and timing of ANC initiation (table 1). Across the 10 countries, women who received more visits and started ANC in the first trimester were more likely to receive all six routine components. However, even among those who received $4+$ visits and started in the first trimester, more than two-fifths of women in each country failed to receive all six routine components. For example, $89.8 \%$ of women in this category in Jordan did not receive all six components. The countries with the highest percentage of women in this category receiving all six routine components were Nigeria, Nepal, Colombia and Haiti (range 52.0\%-54.3\%).

The percentages of women in need of ANC and percentages of ANC users receiving all six routine and all available ANC components are shown in table 2. The percentage of women receiving any ANC was high ( $\geq 85 \%$ in all countries except Nigeria). However, in some countries the coverage with $4+$ visits started in the first trimester was considerably lower. In terms of the content of care, in sub-Saharan Africa the percentage of women who needed ANC care who reported receiving all six routine components was comparable or better than the percentage of women getting $4+$ visits started in the first trimester. This means that even women who had one to three visits and/or initiated ANC later in pregnancy were likely to receive the six routine components. However, for countries in the other regions, the picture was consistently worse. Even among women with many ANC visits $(8+)$ starting in the first trimester, less than two-thirds of women in any country reported receiving all six routine care components. However, the countries with the highest percentage of women with a high number of visits (8+) who reported receiving all care components (Nigeria and Nepal) were those with the lowest levels of overall ANC coverage.

\section{DISCUSSION}

ANC provides a critical opportunity to support pregnant women and ensure that they, and their babies, benefit from effective, good-quality maternal care. This paper responds to an urgent need to understand the provision of ANC to women in LMICs as was done in the MDG-era, and its content, in order to identify gaps and to inform the development of measures focusing on quality of care. We estimated MDG-era ANC indicator of number of ANC visits in 10 countries using recent DHS data. In all countries, the large majority of women had $1+\mathrm{ANC}$ 
Table 1 Receipt of antenatal care (ANC) components (percentage and mean) among women who used ANC, by country

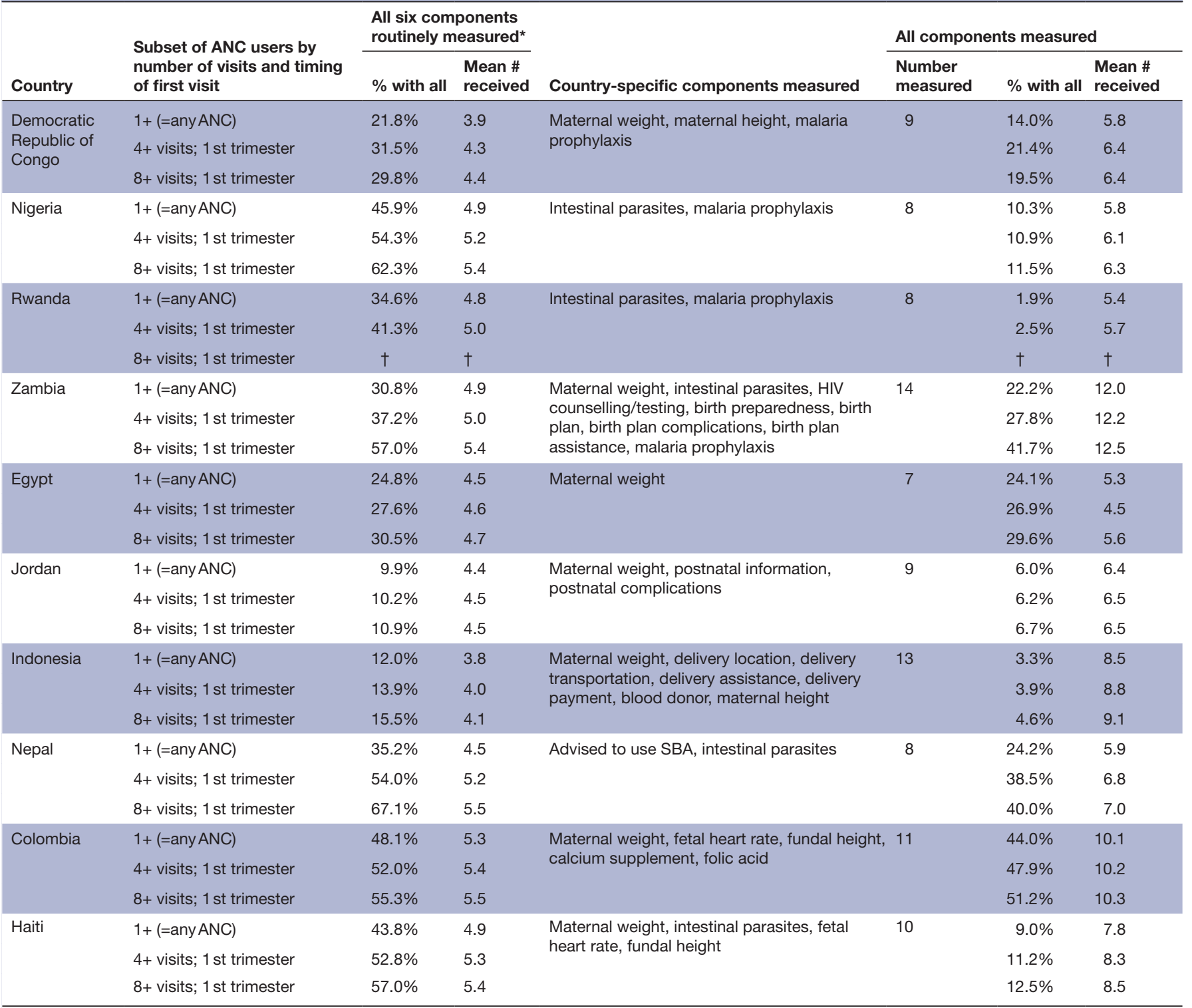

*Six components routinely measured include: blood pressure measured, urine sample taken, blood sample taken, tetanus protection, iron supplementation and receipt of information on potential complications.

†Not estimated in Rwanda due to insufficient sample size.

SBA, skilled birth attendant.

visits (ranging from $65 \%$ in Nigeria to $>98 \%$ in Rwanda, Zambia and Jordan). In all countries, over two-fifths of women had 4+ visits (ranging from $44 \%$ in Rwanda to $94 \%$ in Jordan). On the other hand, the percentage of women who had eight or more visits ranged from $0 \%$ in Rwanda to $69 \%$ in Jordan. Among women who had ANC, the proportions having $4+$ visits and starting in the first trimester ranged from $15 \%$ in DRC to $89 \%$ in Jordan.

In the 10 included countries, between 7 and 14 country-specific ANC components were captured by DHS, of which 6 were common across all surveys. We captured ANC content through understanding the percentage of women who reported receiving each ANC component as captured on surveys, the percentage who reported receiving the six routine components, the percentage of women who reported receiving all components, and an average combined score of available components. We found that receipt of the six routine components varied widely. Across the countries, the most commonly reported component was blood pressure measurement; urine test and information on complications tended to be the least commonly received. Only in Colombia did women with 1+ ANC visits report receiving, on average, more than five of the six routine care components.

We examined 10 countries, which do not represent the variety of experiences and results achieved by LMICs globally, but rather serve as case studies of a range of contexts across four regions to exemplify gaps in ANC coverage, content and quality. In relating these content measures to ANC utilisation patterns, we found that even among women starting ANC in the first trimester and receiving $4+$ visits, the percentage receiving all six 
Table 2 Percentage of women receiving all six routine and all measured antenatal care (ANC) components, by timing of initiation and number of visits, most recent birth

\begin{tabular}{|c|c|c|c|c|c|c|c|c|c|c|}
\hline \multirow{2}{*}{$\begin{array}{l}\text { Region } \\
\text { Country }\end{array}$} & \multicolumn{4}{|c|}{ Sub-Saharan Africa } & \multicolumn{2}{|c|}{ Middle East } & \multicolumn{2}{|l|}{ Asia } & \multicolumn{2}{|c|}{$\begin{array}{l}\text { Latin America and the } \\
\text { Caribbean }\end{array}$} \\
\hline & DRC & Nigeria & Rwanda & Zambia & Egypt & Jordan & Indonesia & Nepal & Colombia & Haiti \\
\hline \multicolumn{11}{|l|}{ ANC visits and timing coverage } \\
\hline $\begin{array}{l}\% \text { of women receiving any ANC } \\
\text { (1+visit) }\end{array}$ & $90.0 \%$ & $65.1 \%$ & $99.2 \%$ & $98.6 \%$ & $91.1 \%$ & $99.1 \%$ & $96.9 \%$ & $85.9 \%$ & $96.7 \%$ & $90.1 \%$ \\
\hline $\begin{array}{l}\% \text { of women receiving } 4+\text { visits started } \\
\text { in first trimester }\end{array}$ & $14.9 \%$ & $24.6 \%$ & $38.6 \%$ & $19.9 \%$ & $80.5 \%$ & $89.0 \%$ & $78.9 \%$ & $44.0 \%$ & $76.1 \%$ & $54.4 \%$ \\
\hline \multicolumn{11}{|c|}{$\%$ receiving all six components routinely measured, among } \\
\hline All women in need of ANC & $19.6 \%$ & $29.9 \%$ & $34.4 \%$ & $30.4 \%$ & $22.6 \%$ & $9.9 \%$ & $11.6 \%$ & $30.3 \%$ & $46.5 \%$ & $39.5 \%$ \\
\hline All women with any ANC (1+ visit) & $21.8 \%$ & $45.9 \%$ & $34.6 \%$ & $30.8 \%$ & $24.8 \%$ & $9.9 \%$ & $12.0 \%$ & $35.2 \%$ & $48.1 \%$ & $43.8 \%$ \\
\hline $\begin{array}{l}\text { Women with } 1+\text { visits started in first } \\
\text { trimester }\end{array}$ & $28.0 \%$ & $51.7 \%$ & $38.1 \%$ & $35.4 \%$ & $27.0 \%$ & $10.1 \%$ & $13.3 \%$ & $44.7 \%$ & $51.5 \%$ & $48.7 \%$ \\
\hline $\begin{array}{l}\text { Women with } 4+\text { visits started in first } \\
\text { trimester }\end{array}$ & $31.5 \%$ & $54.3 \%$ & $41.3 \%$ & $37.2 \%$ & $27.6 \%$ & $10.2 \%$ & $13.9 \%$ & $54.0 \%$ & $52.0 \%$ & $52.8 \%$ \\
\hline $\begin{array}{l}\text { Women with } 8+\text { visits started in first } \\
\text { trimester }\end{array}$ & $29.8 \%$ & $62.3 \%$ & * & $57.0 \%$ & $30.5 \%$ & $10.9 \%$ & $15.5 \%$ & $67.1 \%$ & $55.3 \%$ & $57.0 \%$ \\
\hline Number of all components measured & 9 & 8 & 8 & 14 & 7 & 9 & 13 & 8 & 11 & 10 \\
\hline \multicolumn{11}{|l|}{$\%$ receiving all components measured, among } \\
\hline All women in need of ANC & $12.6 \%$ & $6.7 \%$ & $1.9 \%$ & $21.8 \%$ & $22.0 \%$ & $5.9 \%$ & $3.2 \%$ & $20.8 \%$ & $42.5 \%$ & $8.1 \%$ \\
\hline All women with any ANC (1+ visit) & $14.0 \%$ & $10.3 \%$ & $1.9 \%$ & $22.2 \%$ & $24.1 \%$ & $6.0 \%$ & $3.3 \%$ & $24.2 \%$ & $44.0 \%$ & $9.0 \%$ \\
\hline $\begin{array}{l}\text { Women with } 1+\text { visits started in first } \\
\text { trimester }\end{array}$ & $18.9 \%$ & $10.3 \%$ & $2.3 \%$ & $26.4 \%$ & $26.3 \%$ & $6.2 \%$ & $3.7 \%$ & $31.1 \%$ & $47.4 \%$ & $10.2 \%$ \\
\hline $\begin{array}{l}\text { Women with } 4+\text { visits started in first } \\
\text { trimester }\end{array}$ & $21.4 \%$ & $10.9 \%$ & $2.5 \%$ & $27.8 \%$ & $26.9 \%$ & $5.2 \%$ & $3.9 \%$ & $38.5 \%$ & $47.9 \%$ & $11.2 \%$ \\
\hline $\begin{array}{l}\text { Women with } 8+\text { visits started in first } \\
\text { trimester }\end{array}$ & $19.5 \%$ & $11.5 \%$ & * & $41.7 \%$ & $29.6 \%$ & $6.7 \%$ & $4.6 \%$ & $40.0 \%$ & $51.2 \%$ & $12.5 \%$ \\
\hline \multicolumn{11}{|l|}{ Sample sizes } \\
\hline All women in need of ANC & 9413 & 16464 & 4605 & 7165 & 8599 & 4929 & 9888 & 2912 & 8772 & 3920 \\
\hline All women with any ANC & 8471 & 10717 & 4569 & 7062 & 7836 & 4885 & 9586 & 2500 & 8481 & 3533 \\
\hline $\begin{array}{l}\text { Women with } 1+\text { visits started in the first } \\
\text { trimester }\end{array}$ & 1576 & 2841 & 2582 & 1756 & 6559 & 4468 & 7909 & 1464 & 6586 & 2242 \\
\hline $\begin{array}{l}\text { Women with } 4+\text { visits started in the first } \\
\text { trimester }\end{array}$ & 1259 & 2633 & 1762 & 1403 & 6307 & 4349 & 7566 & 1099 & 6457 & 1922 \\
\hline $\begin{array}{l}\text { Women with } 8+\text { visits started in the first } \\
\text { trimester }\end{array}$ & 103 & 1642 & 2 & 61 & 4744 & 3311 & 5517 & 184 & 3074 & 610 \\
\hline
\end{tabular}

*Not estimated in Rwanda due to insufficient sample size.

DRC, Democratic Republic of Congo.

routine ANC components was low, ranging from $10 \%$ in Jordan to just above $50 \%$ in Nigeria, Nepal, Colombia and Haiti. This suggests that even among women with patterns of care that complied with global recommendations at the time of the surveys, the content of care was inadequate.

The limitations of our analysis stem predominantly from our use of secondary data. Surveys were conducted between 2010 and 2015 and all indicators of ANG use and content rely on women's self-report of events during the pregnancy preceding their most recent live birth. We have no information about patterns of ANC use and content of care for women with pregnancy loss or stillbirth, as these were not collected by the surveys. Women's ANC might have occurred up to 3 years before the survey (and one in Colombia), during which women might forget whether they received a particular component of care. Their reports may be prone to social desirability bias. ${ }^{14}$ The six routine components of ANC care we used are not necessarily the most important components of ANC; rather these six components were the ones that were measured consistently across the included countries and were therefore comparable. Some of these components should be provided at every ANC visit (eg, blood pressure measured), others might only be needed once, but for which good communication and interpersonal skills are needed (eg, information on complications). Others could result from care received prior to the index pregnancy (eg, tetanus toxoid immunisation). For three of the six routine components which should be provided multiple times during a pregnancy, women were only asked whether they received the component 'at least once' during the pregnancy. Additionally, for issues such as counselling about complications, we cannot be sure that women were provided with complete and accurate information. Therefore, our estimates of care coverage 
do not reflect either the correct timing and frequency of these care components across women's ANC visits during pregnancy, or whether the care following on from these components was completed appropriately (eg, What tests were done on the woman's blood sample? Was the woman informed of the results? Was the appropriate action taken based on the findings?). Other important components of ANC, for example, women's experience of care, were not captured at all. These limitations, together with a validation study from China suggesting some over-reporting of ANC care components received, ${ }^{14}$ make it probable that our results overestimate the percentages of women receiving the ANC components.

Our analysis of ANC coverage showed that countries largely achieved high coverage with $1+$ ANC visits, but varied greatly in their adherence to WHO recommendations on the timing of the first visit and content of care. Possible reasons include a lack of policy efforts, prioritisation of expanding ANC coverage to reach all women with $1+$ visit, issues with regulating providers in the non-public sector, or demand-side issues such as acceptability, affordability or physical accessibility of ANC services. It is also surprising that some women started ANC in the first trimester, but did not obtain the recommended number of $4+$ visits, as these women ( $\geq 5 \%$ in 4 of the 10 countries, reaching a maximum of $18 \%$ in Rwanda) were clearly able to overcome any initial demand-side barriers. This pattern might stem from context-specific processes, such a requirement for one ANC visit to obtain a delivery booking card, or might indicate a woman's experience of poorquality ANC in an early visit possibly coupled with an inability to switch to an alternative care provider led them to curtail their number of visits. Notwithstanding such explanations, it will be crucial for countries to focus on retaining women in ANC by understanding and addressing barriers to initial, early access and continued use as well as understanding factors influencing provision of good-quality care by providers. ${ }^{15} 16$ We also showed inequities in the use of ANC, where substantial proportions of women did not receive any ANC, while high percentages of women reported well over eight visits. Nigeria was an extreme example of this pattern; it had the lowest percentage of women using any ANC and the highest percentage of women receiving 20+ visits. This pattern might relate to lack of accessibility of public sector ANC and considerable utilisation of private sector ANC, predominantly by more educated, wealthier women. ${ }^{1718}$

Our examination of ANCcontent showed various patterns suggesting context-specific drivers of high and low coverage of specific ANC components. It is interesting to compare the relatively low percentages of women with $4+$ visits/start in first trimester receiving all six routine components to the relatively high country means of the number of components received (the lowest was 4.0 in Indonesia and the highest 5.4 in Colombia). This means that, on average, countries were only one or two components short of providing all six routine components. In the six countries with means of at least five components (on average, one component too few), all had one particular ANC component lagging behind in coverage (information on complications in Nigeria and Haiti; urine test in Rwanda and Zambia; blood test in Nepal; and tetanus in Colombia). Such patterns also emerge for the four countries which averaged between four and five components, where focusing on one to two specific-care components would have greatly enhanced the percentage of women with all routine components. The mapping of the routine components with the lowest coverage showed interesting within-region consistencies in the care components in greatest need of improvement: sub-Saharan Africa (urine test and information on complications), Middle East (information on complications, iron supplementation and tetanus), Asia (urine test and blood test) and Latin America and the Caribbean (tetanus and information on complications).

In terms of individual components, blood pressure measurement had the highest coverage across the countries ( $>80 \%$ of women with $4+$ visitsand starting in the first trimester in all 10 countries). This might relate to the need to carry out this practice at every ANC visit, therefore increasing women's chance of having had their blood pressure measured at least once during ANC and recalling such measurement; it shows high effective coverage even among women with few visits (one to three) and those starting ANC in the second trimester of pregnancy or later. However, a recent study linking DHS and facility readiness in sub-Saharan Africa showed particularly poor ability to manage hypertensive disease in pregnancy, indicating that measurement of blood pressure alone may not be adequate for quality care. ${ }^{19}$ Coverage of urine tests, which should also be performed on multiple occasions during ANC, was lower than blood pressure measurement, possibly because it relied on the availability of consumable supplies or functional laboratories. Additionally, in the sub-Saharan countries we analysed, the coverage of blood tests was higher than for urine tests, which might reflect a strong commitment to HIV testing or measurement of anaemia during ANC. A recent study in Mozambique showed that screening for high blood pressure, proteinuria and anaemia was much less common compared with HIV testing and the provision, at point of care, of supplies for evidence-based practices packaged in kits resulted in a vast improvement in these practices. ${ }^{20}$

On the other hand, coverage with receipt of information on complications was very low in most countries, despite the fact that communicating such information requires no supplies or equipment, the only cost being staff time. In light of the importance of ANC in the pathways to appropriate delivery care, ${ }^{21-23}$ and the fact that it is also identified a critical component by women themselves,${ }^{24}$ this component of ANC requires immediate improvement. In countries where this low coverage is linked to staff shortages or facility overcrowding, alternative provision models could be explored, such as group ANC or task-sharing. ${ }^{25}$ Tetanus 
toxoid coverage was surprisingly low in the two Middle Eastern countries, considering that both have eliminated neonatal tetanus. This may suggest a diminution of focus after achieving elimination goals, and warrants further investigation. ${ }^{26} 27$

We showed a large gap between the number of ANC visits $(1+$ and $4+)$ and measures of ANC content. This issue has been highlighted by studies using other data sources. ${ }^{28-30}$ Our findings bolster studies highlighting suboptimal quality of ANC in LMICs in general, ${ }^{31}{ }^{32}$ and among socioeconomically vulnerable groups of women in particular. ${ }^{18} 33$ We found evidence, particularly in countries outside sub-Saharan Africa, suggesting that the focus on the number of ANC visits may have been to the detriment of ensuring effective coverage. Countries with larger variations in the number of ANC visits (eg, Egypt, Jordan, Indonesia) were less likely to reach high coverage with all six routinely measured components of care, despite their higher overall median number of visits. This was true when ANC content was assessed among all women with need for ANC and among women who received 4+ ANC visits and started ANC in the first trimester of pregnancy.

In certain contexts, some women might genuinely require higher number of ANC visits, reflecting a disease burden and individual needs. However, the variability in number of visits seen in our analysis might also stem from a lack of equity and standards. We note that the three countries with the worst coverage of ANC content-despite a high median number of visits and high utilisation of any ANCEgypt, Jordan and Indonesia, all have health systems highly reliant on private provision of maternal services in general and of ANC in particular. ${ }^{17}$ Understanding of private sector provision-including use of standardised guidelines, dual clinician practice in public/private sectors and incentive structures for high number of visits created by the use of packaged deals-is needed in order to identify opportunities to improve care quality. Unchecked and excessive use of technologies such as prenatal testing and ultrasound beyond what is recommended in the new ANC guidelines might increase the median number of ANC visits in LMICs in the near future, but such population-level trends could mask large socioeconomic inequalities in access to and use of good-quality ANC with appropriate content of care as well as lead to unnecessary healthcare expenditure, including out of pocket. $^{34} 35$

\section{CONCLUSION}

Global coverage with ANC visits starting in the first trimester of pregnancy increased by $43 \%$ between 1990 and 2013 to a level of $58.6 \% .^{36}$ Research shows that the quality of ANC matters for the survival of the mother and child, ${ }^{37} 38$ carries crucial importance in the pathway to women's use of skilled delivery care ${ }^{21-23}$ and can contribute to a positive pregnancy experience. ${ }^{24}$ The 2016 WHO ANC recommendations shifted the focus from coverage to quality and terminology from ANC visits to ANC contacts, implying an active connection between a pregnant woman and her healthcare provider(s). A 'contact' can take place at the facility or community level, and may include context-specific recommendations depending on the health system (eg, task shifting some responsibilities to community-based workers) and on burden of disease (eg, prevention of malaria during pregnancy) ${ }^{39}$ WHO is currently developing a monitoring and evaluation framework based on the new ANG recommendations that will be finalised by the end of 2018, and which will include content of care. Not any one tool/method or indicator will capture all dimensions of care (frequency, initiation, quality), and several sources should ideally be used in order to triangulate results of coverage and quality, in addition to gathering other data helpful with identification of problems and potential solutions (such as supply stock-outs, etc) (box 1).

In light of this paradigm shift, global and national progress towards universal coverage with good-quality ANC can only be monitored by rethinking which content of care and which indicators are measured and why, ensuring that the tools and data sources used to capture these indicators are appropriate, valid, reliable and complementary, and that mechanisms exist to ensure

Box 1 Implications for data sources-antenatal care (ANC) measurement

Population-based surveys relying on women's report (Demographic and Health Surveys, Multiple Indicator Cluster Survey, etc) should capture care content that is in line with evidence-based WHO recommendations, appropriate for the setting and carries acceptable measurement validity given the variable timing of provision for the numerous care components during pregnancy. In addition, identifying such indicators of ANC content could spur national action towards improving quality of ANC content in line with the truism 'what gets measured gets done'. The question then is-given the limited space on surveys, what are the most important ANC components to measure on such surveys? The following are some examples of possible questions or areas of exploration:

Was the woman physically examined by a provider? (eg, did someone touch your belly/measure the belly?)

- Did anyone tell you about your estimated delivery date?

- Were you informed of the results of the blood/urine sample taken?

- How can women's experience of care related to respect, communication and support during ANC be measured?

- Providing a 'don't know' response option for receipt of ANC care components could minimise error due to social desirability. Other data sources could also be explored as routine health management information system data such as District health information system 2 (DHIS2) and digital registries and integrated mHealth strategies become more common. ${ }^{40}$ Provider surveys (Service Provision Assessment (SPA)/Service Availability and Readiness Assessment (SARA)), routine supervision visits/ANC consultation observations, client exit surveys, mystery client visits, and women-held ANC cards are other methods for measuring different aspects of ANC for monitoring and improvement purposes. 
efficient and effective action to improve care quality on the basis of these indicators.

Acknowledgements The authors thank Ms Emily Wilson for assistance with preparing the tables. The authors also thank the DHS program for collecting and making available the data and the women who participated in these surveys.

Contributors OMRC had the original idea for this analysis. OMRC, LB and ÖT designed the analyses. LB conducted the data analysis and drafted tables and figures. OMRC, LB, ACM and ÖT participated in interpreting the results, and in drafting and commenting on the paper.

Funding The research in this manuscript was supported by funding from MSD through its MSD for Mothers programme. Funding was used for general financial support, including staff salaries, travel and overheads.

Disclaimer The content of this report is solely the responsibility of the authors and does not represent the official views of MSD. MSD for Mothers is an initiative of Merck \& Co, Inc, Kenilworth, New Jersey, USA. The content of this article is solely the responsibility of the authors and does not necessarily represent the official views of the institutions to which the authors are affiliated.

Competing interests LB reports receiving a research grant from Merck Sharp and Dohme (MSD) through its MSD for Mothers programme.

Patient consent Not required.

Ethics approval Research Ethics Committee of the London School of Hygiene and Tropical Medicine.

Provenance and peer review Not commissioned; externally peer reviewed.

Data sharing statement The data used in this article are available to download for research purposes upon registration; www.dhsprogram.com.

Open Access This is an Open Access article distributed in accordance with the Creative Commons Attribution Non Commercial (CC BY-NC 4.0) license, which permits others to distribute, remix, adapt, build upon this work non-commercially, and license their derivative works on different terms, provided the original work is properly cited and the use is non-commercial. See: http://creativecommons.org/ licenses/by-nc/4.0/

(c) Article author(s) (or their employer(s) unless otherwise stated in the text of the article) 2018. All rights reserved. No commercial use is permitted unless otherwise expressly granted.

\section{REFERENCES}

1. Alkema L, Chou D, Hogan D, et al. Global, regional, and national levels and trends in maternal mortality between 1990 and 2015, with scenario-based projections to 2030: a systematic analysis by the UN Maternal Mortality Estimation Inter-Agency Group. Lancet 2016;387:462-74.

2. Blencowe $\mathrm{H}$, Cousens $\mathrm{S}$, Jassir FB, et al. National, regional, and worldwide estimates of stillbirth rates in 2015, with trends from 2000: a systematic analysis. Lancet Glob Health 2016;4:e98-e108.

3. World Health Organization. Global Strategy for Women's, Children's and Adolescents Health (2016-2030). Geneva: WHO, 2015.

4. United Nations. Sustainable development goals. http://www.un.org/ sustainabledevelopment/sustainable-development-goals/ (accessed 14 Nov 2017).

5. Kuhnt J, Vollmer S. Antenatal care services and its implications for vital and health outcomes of children: evidence from 193 surveys in 69 low-income and middle-income countries. BMJ Open 2017;7:e017122.

6. Graham W, Woodd S, Byass P, et al. Diversity and divergence: the dynamic burden of poor maternal health. Lancet 2016;388:2164-75.

7. World Health Organization. WHO recommendations on antenatal care for a positive pregnancy experience. Geneva: WHO, 2016

8. United Nations. Official List of MDG indicators. $2008 \mathrm{http} / / / \mathrm{mdgs}$. un.org/unsd/mdg/Host.aspx?Content=Indicators/OfficialList.htm (accessed 14 Nov 2017).

9. Moran AC, Jolivet RR, Chou D, et al. A common monitoring framework for ending preventable maternal mortality, 20152030: phase I of a multi-step process. BMC Pregnancy Childbirth 2016;16:250

10. Hodgins S, D'Agostino A. The quality-coverage gap in antenatal care: toward better measurement of effective coverage. Science and Practice: Global Health, 2014.
11. Ng M, Fullman N, Dieleman JL, et al. Effective coverage: a metric for monitoring Universal Health Coverage. PLoS Med 2014;11:e1001730.

12. Saad-Haddad G, DeJong J, Terreri N, et al. Patterns and determinants of antenatal care utilization: analysis of national survey data in seven countdown countries. J Glob Health 2016;6:010404.

13. World Health Organization. WHO Antenatal care randomized trial: Manual for the implementation of the new model. Geneva: UNDP/ UNFPA/WHO/World Bank Special Programme of Research, Development and Research Training in Human Reproduction, 2002.

14. Liu L, Li M, Yang L, et al. Measuring coverage in MNCH: a validation study linking population survey derived coverage to maternal, newborn, and child health care records in rural China. PLoS One 2013;8:e60762.

15. Downe S, Finlayson K, Tunçalp Özge, et al. Factors that influence the uptake of routine antenatal services by pregnant women: a qualitative evidence synthesis. Cochrane Database Syst Rev 2016;24.

16. Downe S, Finlayson K, Tuncalp O, et al. Factors that influence the provision of good-quality routine antenatal services: a qualitative evidence synthesis of the views and experiences of maternity care providers. Cochrane Database of Systematic Reviews 2017;8.

17. Campbell OM, Benova L, MacLeod D, et al. Family planning, antenatal and delivery care: cross-sectional survey evidence on levels of coverage and inequalities by public and private sector in 57 low- and middle-income countries. Trop Med Int Health 2016;21:486-503.

18. Powell-Jackson T, Macleod D, Benova L, et al. The role of the private sector in the provision of antenatal care: a study of Demographic and Health Surveys from 46 low- and middle-income countries. Trop Med Int Health 2015;20:230-9.

19. Kanyangarara M, Munos MK, Walker N. Quality of antenatal care service provision in health facilities across sub-Saharan Africa: Evidence from nationally representative health facility assessments. $J$ Glob Health 2017;7:021101.

20. Betrán AP, Bergel E, Griffin S, et al. Provision of medical supply kits to improve quality of antenatal care in Mozambique: a steppedwedge cluster randomised trial. Lancet Glob Health 2018;6:e57-e65.

21. Chukwuma A, Wosu AC, Mbachu C, et al. Quality of antenatal care predicts retention in skilled birth attendance: a multilevel analysis of 28 African countries. BMC Pregnancy Childbirth 2017;17:152.

22. Adjiwanou V, Legrand T. Does antenatal care matter in the use of skilled birth attendance in rural Africa: a multi-country analysis. Soc Sci Med 2013;86:26-34.

23. Benova L, Macleod D, Radovich E, et al. Should I stay or should go?: consistency and switching of delivery locations among new mothers in 39 Sub-Saharan African and South/Southeast Asian countries. Health Policy Plan 2017;32:1294-308.

24. Downe S, Finlayson K, Tunçalp Ö, et al. What matters to women: a systematic scoping review to identify the processes and outcomes of antenatal care provision that are important to healthy pregnant women. BJOG 2016;123:529-39.

25. Mazzoni SE, Carter EB. Group prenatal care. Am J Obstet Gynecol 2017;216:552-6.

26. Khan R, Vandelaer J, Yakubu A, et al. Maternal and neonatal tetanus elimination: from protecting women and newborns to protecting all. Int J Womens Health 2015;7:171-80.

27. Hassan AM, Shoman AE, Abo-Elezz NF, et al. Tetanus vaccination status and its associated factors among women attending a primary healthcare center in Cairo governorate, Egypt. J Egypt Public Health Assoc 2016;91:127-34.

28. Kyei NN, Chansa C, Gabrysch S. Quality of antenatal care in Zambia: a national assessment. BMC Pregnancy Childbirth 2012;12:151.

29. Marchant T, Tilley-Gyado RD, Tessema T, et al. Adding content to contacts: measurement of high quality contacts for maternal and newborn health in Ethiopia, north east Nigeria, and Uttar Pradesh, India. PLoS One 2015;10:e0126840.

30. Heredia-Pi I, Servan-Mori E, Darney BG, et al. Measuring the adequacy of antenatal health care: a national cross-sectional study in Mexico. Bull World Health Organ 2016;94:452-61.

31. Yeoh PL, Hornetz K, Ahmad Shauki NI, et al. Assessing the extent of adherence to the recommended antenatal care content in Malaysia: room for improvement. PLoS One 2015;10:e0135301.

32. Boller C, Wyss K, Mtasiwa D, et al. Quality and comparison of antenatal care in public and private providers in the United Republic of Tanzania. Bull World Health Organ 2003;81:116-22.

33. Owolabi OO, Wong KLM, Dennis ML, et al. Comparing the use and content of antenatal care in adolescent and older first-time mothers in 13 countries of west Africa: a cross-sectional analysis of Demographic and Health Surveys. Lancet Child Adolesc Health 2017;1:203-12. 
34. Bashour H, Hafez R, Abdulsalam A. Syrian women's perceptions and experiences of ultrasound screening in pregnancy: implications for antenatal policy. Reprod Health Matters 2005:13:147-54

35. Benova L, Campbell OM, Ploubidis GB. A mediation approach to understanding socio-economic inequalities in maternal healthseeking behaviours in Egypt. BMC Health Serv Res 2015;15:1.

36. Moller AB, Petzold M, Chou D, et al. Early antenatal care visit: a systematic analysis of regional and global levels and trends of coverage from 1990 to 2013. Lancet Glob Health 2017;5:e977-e983.
37. Hodgins S, Tielsch J, Rankin K, et al. A new look at care in pregnancy: simple, effective interventions for neglected populations. PLoS One 2016;11:e0160562.

38. Amoakoh-Coleman M, Klipstein-Grobusch K, Agyepong IA, et al. Provider adherence to first antenatal care guidelines and risk of pregnancy complications in public sector facilities: a Ghanaian cohort study. BMC Pregnancy Childbirth 2016;16:369.

39. Tunçalp Ö, Pena-Rosas JP, Lawrie T, et al. WHO recommendations on antenatal care for a positive pregnancy experience-going beyond survival. BJOG 2017;124:860-2.

40. Mehl G, Labrique A. Prioritizing integrated $m$ Health strategies for universal health coverage. Science 2014;345:1284-7. 